\title{
On the Probability of Real Roots in a Quadratic Equation with Coefficients as i.i.d $\mathcal{U}(-\boldsymbol{\theta}, \boldsymbol{\theta})$ Variates
}

\author{
Subhomoy Haldar, Soubhik Chakraborty* \\ Department of Mathematics, Birla Institute of Technology, Mesra, Ranchi-835215, India.
}

How to cite this paper: Subhomoy Haldar, Soubhik Chakraborty. (2021) On the Probability of Real Roots in a Quadratic Equation with Coefficients as i.i.d $\mathcal{U}(-\theta, \theta) \mathrm{Va}-$ riates. Journal of Applied Mathematics and Computation, 5(1), 48-55.

DOI: 10.26855/jamc.2021.03.006

Received: January 29, 2021

Accepted: February 25, 2021

Published: March 12, 2021

${ }^{*}$ Corresponding author: Soubhik Chakraborty, Department of Mathematics, Birla Institute of Technology, Mesra, Ranchi-835215, India.

Email: soubhikc@yahoo.co.in

\begin{abstract}
In this paper, we seek to find out the probability of obtaining real roots of a quadratic equation $A X^{2}+B X+C=0$, with $A \neq 0$, when the coefficients are independent, identically distributed uniform variates. The exact value of the roots can be obtained from the coefficients and the discriminant indicates if the roots are real or imaginary. Here, we consider the uniform distribution $U(-\theta, \theta)$ and find the probability of obtaining a real root to be $62.7 \%$. This is done through simplification of the problem, analysis of the probability distribution of $\mathrm{B}^{2}$ for both $\mathcal{U}(-1,1)$ and $\mathcal{U}(0,1)$, and final evaluation using conditional probability. Calculations are simplified by the fact that $\mathrm{B}^{2} \geq 4 \mathrm{AC}$ is always true when $\mathrm{AC} \leq 0$. We leverage on the fact that the probability of obtaining real roots when coefficients are sampled from $\mathcal{U}(0, \theta)$ is $25.4 \%$. We verify the result experimentally through Monte Carlo simulation and present the desired supporting data accordingly.
\end{abstract}

\section{Keywords}

Quadratic Equation, Continuous Uniform Distribution, Probability, Monte Carlo Simulation

\section{Introduction}

We consider the general form of a quadratic equation as $\mathrm{A} X^{2}+\mathrm{BX}+\mathrm{C}=0, \mathrm{~A} \neq 0$, where $\mathrm{A}, \mathrm{B}$, and $\mathrm{C}$ are called the coefficients. We limit the discussion to real-valued coefficients only. This is because random variables are real-valued, and we want to learn about the nature of the roots when the coefficients are sampled from well-known distributions. The quadratic formula used to calculate the roots exactly is:

$$
\mathrm{x}=\frac{-B \pm \sqrt{B^{2}-4 A C}}{2 A}
$$

The nature of the roots is determined easily using the determinant $\mathrm{D}=B^{2}-4 \mathrm{AC}$. If $\mathrm{D}>0$, the roots are real and unequal. If $\mathrm{D}=0$, the roots are real and equal. If $\mathrm{D}<0$, the roots are imaginary and occur in conjugate pairs, that is, of the form $\mathrm{p}+\mathrm{iq}$ and $\mathrm{p}-\mathrm{iq}$, where $\mathrm{i}=\sqrt{-1}$ is the imaginary number and $\mathrm{p}, \mathrm{q}$ are real. We shall be concentrating in the probability of obtaining a real root when the three coefficients $\mathrm{A}, \mathrm{B}$, and $\mathrm{C}$ are sampled from the continuous uniform distribution $U(-1,+1)$. This is because, given a $\theta>0$, it suffices to consider the interval $(-1,1)$ instead of $(-\theta, \theta)$ as the values of the roots do not change if we divide the coefficients by $\theta$.

Continuing our discussion on the value of the discriminant $\mathrm{D}$, if $\mathrm{D}$ is negative, $\sqrt{D}$ is imaginary and so are the roots. 
We have $\mathrm{E}[A]=\mathrm{E}[C]=0$ and $\mathrm{E}\left[B^{2}\right]=\frac{1}{3}$ (proved in the appendix of the paper). So $\mathrm{E}[D]=\frac{1}{3}-4 \times 0=\frac{1}{3}$. Hence, on average, our roots will be real and separated by $\frac{2}{A \sqrt{3}}$. Note that $A \neq 0$.

\section{Theoretical Results}

The current distribution under consideration, that is, $\mathcal{U}(-1,1)$ shares a lot of similarities with the distribution $U(0,1)$ which is generally considered in such studies. Therefore, we shall try to find symmetries and reduce the problem into the general case whenever possible. Our analysis is inspired from [1].

\subsection{The CDF of $B^{2}$ is unchanged}

The probability distribution function (PDF) $f_{X}(x)$ of a uniform distribution is defined in [2]. We use that definition to obtain the cumulative frequency distribution (CDF) $F_{X}(x)$.

Theorem 2.1.1: The distribution of $B^{2}$ is the same regardless of $B$ being sampled from $\mathcal{U}(0,1)$ or $U(-1,1)$.

Proof: We define $X \sim U(0,1)$ and $Y \sim U(-1,+1)$. We then have

$$
\begin{gathered}
F_{X}(x)=\mathrm{P}(X \leq x)=F_{X}(x)= \begin{cases}0, & x<0 \\
x, & 0 \leq x \leq 1 \\
1, & x>1\end{cases} \\
F_{Y}(y)=\mathrm{P}(Y \leq y)=F_{Y}(y)=\left\{\begin{array}{cl}
0, & x<-1 \\
\frac{y+1}{2,} & -1 \leq x \leq+1 \\
1, & x>+1
\end{array}\right.
\end{gathered}
$$

We now calculate the CDFs for $X^{2}$ and $Y^{2}$.

$$
\begin{aligned}
& F_{X^{2}}(x)=P\left(X^{2} \leq x\right)=P(|X| \leq \sqrt{x})=\left\{\begin{array}{cl}
0, & x<0 \\
\sqrt{x}, & 0 \leq x \leq 1 \\
1, & x>1
\end{array}\right. \\
& F_{Y^{2}}(y)=\mathrm{P}\left(Y^{2} \leq y\right)=\mathrm{f}(x)=\left\{\begin{array}{rr}
0, & y<0 \\
P(|Y| \leq \sqrt{y}), & y \geq 0
\end{array}\right.
\end{aligned}
$$

Notice that when $Y \sim \mathcal{U}(-1,+1)$, we have $\mathrm{P}(Y \leq y)=\left\{\begin{array}{c}0, y<-1 \\ \frac{y+1}{2},-1 \leq y \leq+1 \\ 1, y>+1\end{array}\right.$

This is because the allowed region for $Y$ includes $(-1,0)$ and $(0, y)$.

Similarly, $\mathrm{P}(Y \geq y)=\left\{\begin{array}{c}1, y<-1 \\ \frac{1-y}{2},-1 \leq y \leq+1 \\ 0, y>+1\end{array}\right.$

Here the allowed regions are $(-y, 0)$ and $(0,1)$.

Hence, we have

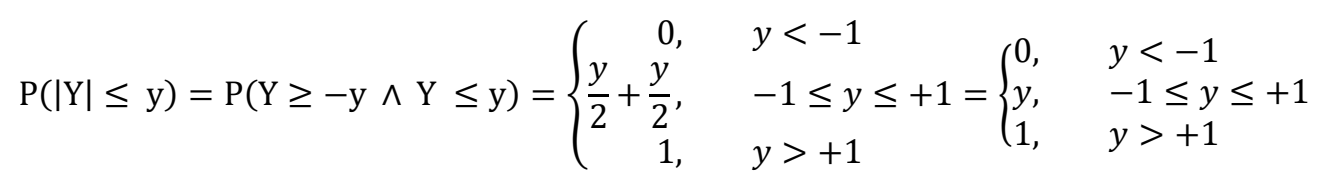

because the allowed regions are $(-y, 0)$ and $(0, y)$. Consequently, we obtain the final expression:

$$
F_{Y^{2}}(y)=\left\{\begin{aligned}
0, & y<0 \\
\sqrt{y}, & 0 \leq y \leq 1 \\
1, & y>1
\end{aligned}\right.
$$

Verification: The CDFs for $X^{2}$ and $Y^{2}$ are valid (and equal) because:

1. $\lim _{x \rightarrow-\infty} F_{X^{2}}(x)=\lim _{x \rightarrow 0} F_{X^{2}}(x)=0$ and $\lim _{y \rightarrow-\infty} F_{Y^{2}}(y)=\lim _{y \rightarrow 0} F_{Y^{2}}(y)=0$

2. $\lim _{x \rightarrow+\infty} F_{X^{2}}(x)=\lim _{x \rightarrow 1} F_{X^{2}}(x)=1$ and $\lim _{y \rightarrow+\infty} F_{Y^{2}}(y)=\lim _{y \rightarrow 1} F_{Y^{2}}(y)=1$

3. $\frac{d}{d x} F_{X^{2}}(x)=\frac{d}{d x} F_{Y^{2}}(x)=\frac{1}{2 \sqrt{x}}$ which is greater than 0 for $x \in(0,1)$ and monotonically decreasing. This means $F_{X^{2}}(x)$ and $F_{Y^{2}}(y)$ are monotone and non-decreasing. 
The CDF expressions for $X^{2}$ and $Y^{2}: F_{X^{2}}(x)$ and $F_{Y^{2}}(y)$ are equivalent. Hence, the probability distribution of $B^{2}$ is the same regardless of whether $B$ is sampled from $U(0,1)$ or $\mathcal{U}(-1,1)$.Q.E.D.

This result is important in the next part of the problem, helping in reduction of the larger case of $U(-1,1)$ into the smaller case of $\mathcal{U}(0,1)$ whose result is known.

\subsection{Conditioning on $\mathrm{A}$ and $\mathrm{C}$}

We have $\mathrm{A}, \mathrm{C} \sim \mathcal{U}(-1,+1)$. We can consider 4 quadrants based on the signs of $A$ and $C$ analogous to the quadrants on the $X Y$ plane. We note that in the second quadrant $(A<0, C \geq 0)$ and fourth quadrant $(A>0, C \leq 0)$, when the product $A C \leq 0$, we always have $D=B^{2}-4 A C \geq 0$. Similarly, in the first quadrant $(A>0, C \geq 0)$ and third quadrant $(A<0, C \leq 0)$ we have $A C \geq 0$. Therefore, it is comparable to the case when $\mathrm{A}, \mathrm{C} \sim U(0,1)$.

In theorem 2.1.1, we have proved that the probability distribution of $B^{2}$ remains the same regardless of where we sample B from (out of $U(0,1)$ or $U(-1,+1)$ ). The probability of obtaining a real-root with A, B, C as i.i.d $U(0,1)$ variates is $\frac{1}{36}(5+6 \cdot \log (2)) \approx 0.2544134190$ as shown in [3]. Also, $P(A C \leq 0)=P(A C \geq 0)=\frac{1}{2}$ because all the four quadrants have the same area.

Combining all these findings together, we have

$$
\begin{aligned}
P(D \geq 0)=\mathrm{P}(D & \geq 0 \wedge A C \geq 0) \mathrm{P}(A C \geq 0)+\mathrm{P}(D \geq 0 \wedge A C \leq 0) \mathrm{P}(A C \leq 0) \\
& =\frac{1}{2} P(D \geq 0 \wedge A C \geq 0)+\frac{1}{2} P(D \geq 0 \wedge A C \leq 0) \\
& =\frac{1}{2} P\left(B^{2}-4 A C \geq 0 \wedge A C \geq 0\right)+\frac{1}{2} P\left(B^{2}-4 A C \geq 0 \wedge A C \leq 0\right) \\
& =\frac{1}{2} \times\left(\frac{1}{36}(5+6 \cdot \log (2))\right)+\frac{1}{2} \times 1=\frac{\log (2)}{12}+\frac{41}{72} \approx 0.6272067095
\end{aligned}
$$

Hence, the probability of obtaining a real root is about $62.7 \%$. An alternate approach using Iverson brackets can be found in [4].

\section{Experimental Results}

We perform two sets of simulations; the first for verifying that the probability distribution of $B^{2}$ remains unchanged when $B$ is sampled from $\mathcal{U}(-1,+1)$ instead of $\mathcal{U}(0,1)$. The second is a Monte Carlo simulation method (a combination of probability theory and sampling technique) to empirically obtain the probability of a real root. See [5] for further literature.

The simulations are performed using Python in separate Jupyter Notebooks. Link to the repository containing the notebooks is provided in the appendix.

\subsection{Empirical and Theoretical Graphs for $B^{2}$}

The steps performed for this experiment are:

1. Sampling values from $U(0,1)$ and recording the cumulative frequency for $B^{2}$.

2. Sampling values from $U(-1,1)$ and recording the cumulative frequency for $B^{2}$.

3. Plotting the theoretical CDF for $B^{2}$ for comparison.

Figure 1 to Figure 3 summarize our results.

The number of samples was set at 20,000 for all the simulations. On a system with reasonable specifications, the inexpensive calculations can be performed in a few seconds. The first 2 figures are almost identical. The third also matches the first two closely, suggesting that the theoretical expression calculated is correct, and indeed, the probability distribution of $B^{2}$ does not change when $B$ is sampled from $U(-1,1)$ instead of $\mathcal{U}(0,1)$.

\subsection{Simulation Data for Probability of $B^{2} \geq 4 A C$}

For the second set of simulations, we perform the following steps:

1. Given an upper bound $\theta$ for the distribution $\mathcal{U}(-\theta, \theta)$, we perform a pre-determined number of trials.

2. Each trial gives us values for $A, B$, and $C$. We use these to calculate the discriminant and check if it is non-negative. Alternatively, we can see if $B^{2} \geq 4 A C$.

3. In case the criterion is met, we mark that trial as a success and continue.

4. After all the trials are complete, we calculate the probability of a real root for this value of $\theta$ as the fraction of 
successful trials over the total number of trials.

5. We perform all the previous steps again for different values of $\theta$.

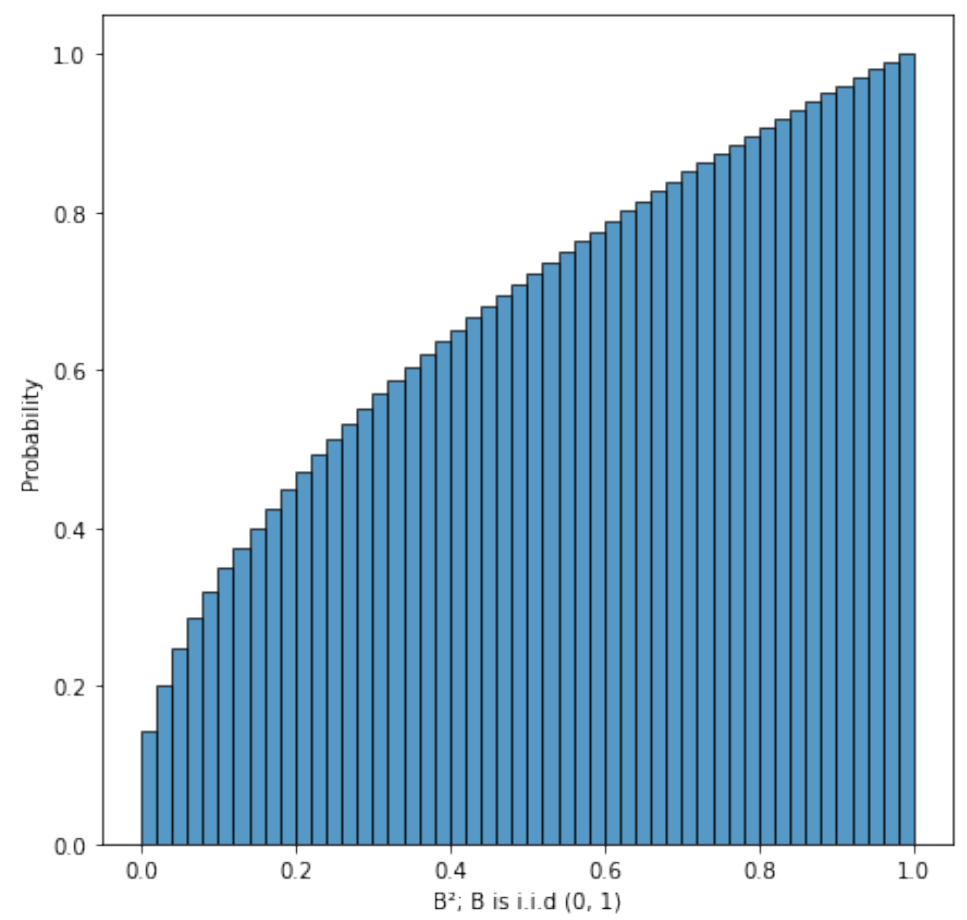

Figure 1. Cumulative frequency for $\mathrm{B}^{2}$ with $\mathrm{B} \sim \mathcal{U}(0,1)$.

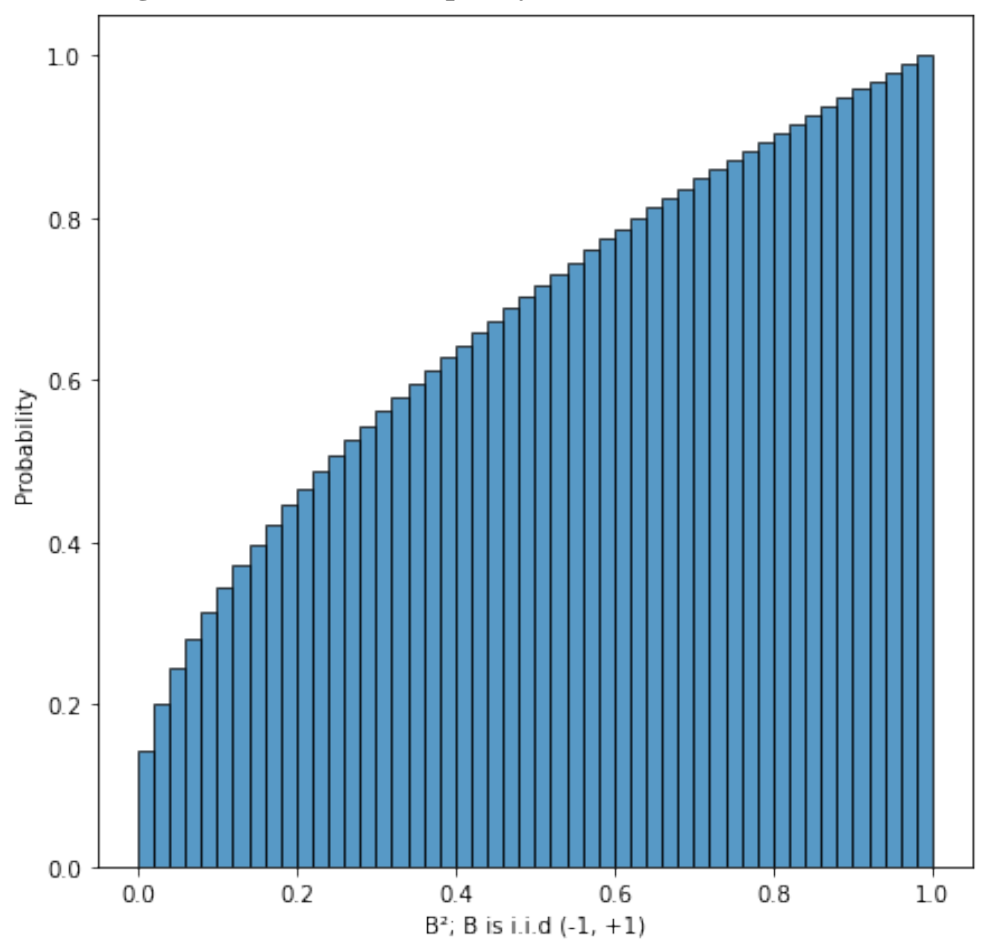

Figure 2. Cumulative frequency for $B^{2}$ with $B \sim \mathcal{U}(-1,1)$. 


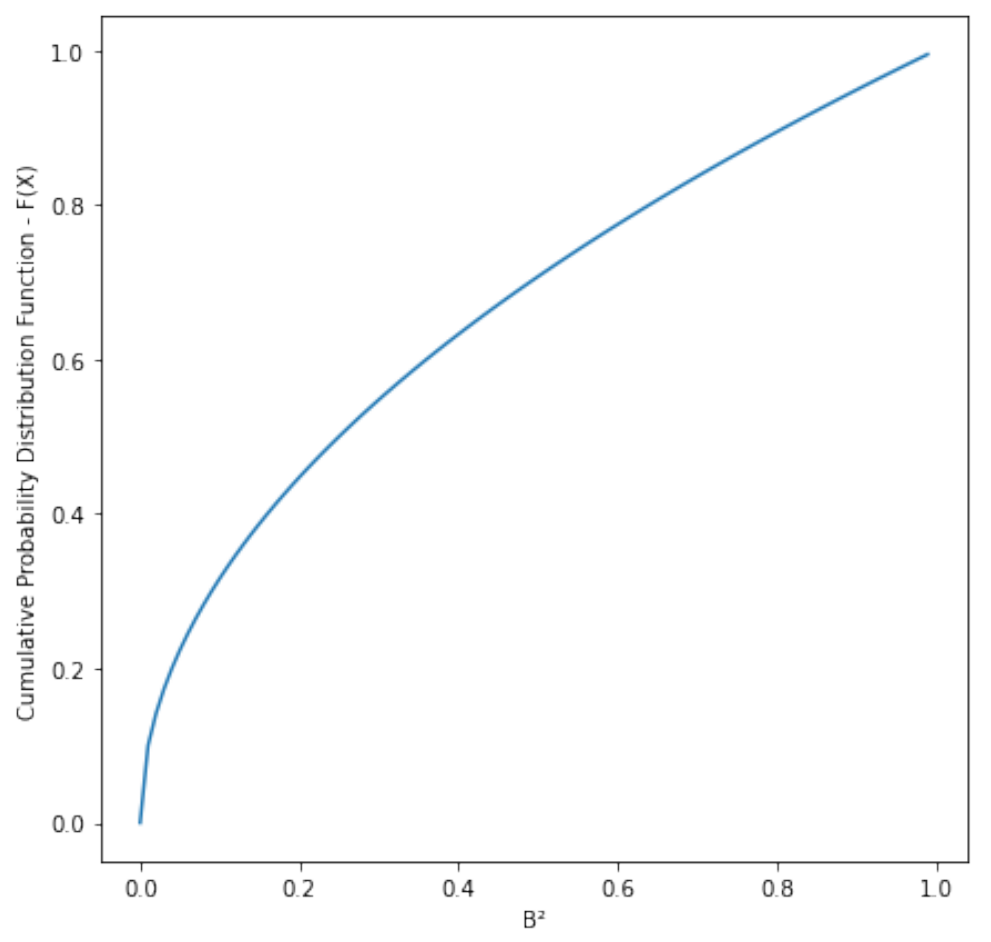

Figure 3. Plot of theoretical CDF of $\mathbf{B}^{2}$.

The probabilities for all the values of $\theta$ tested are tabulated. For each value of $\theta$, the number of trials was set to 20,000 again. Two different tables, Table 1 and Table 2, are prepared: one for small values of $\theta$, and the other for large values respectively.

From Table 1, the mean probability is $62.51 \%$ with a standard deviation of $0.33 \%$, while from Table 2, the mean probability is $62.72 \%$ with a standard deviation of $0.35 \%$. We have therefore, endorsed the correctness of the theoretical value of $62.7 \%$ experimentally.

\section{Discussion}

This study was concerned with the probability of real roots when the coefficients are sampled independently from a uniform distribution of the form $U(-\theta, \theta)$. The empirical probability for the same can be easily computed for different distributions. The only changes needed are to the sampling function. The theoretical analysis could be nontrivial which would make the simulation results even more interesting. It is also of interest to consider the more general $\mathcal{U}(\alpha, \beta)$ with the provision of $\alpha$ being negative.

Table 1. Table of observations for small $\theta$

\begin{tabular}{ccc}
\hline Theta( $(\boldsymbol{)})$ & Favourable Outcomes & Probability \\
\hline 1 & 12,556 & 0.62780 \\
2 & 12,312 & 0.61560 \\
3 & 12,492 & 0.62460 \\
4 & 12,593 & 0.62965 \\
5 & 12,466 & 0.62330 \\
6 & 12,534 & 0.62670 \\
7 & 12,479 & 0.62395 \\
8 & 12,469 & 0.62345 \\
9 & 12,532 & 0.62660 \\
\hline
\end{tabular}




\begin{tabular}{lll}
\hline 10 & 12,522 & 0.62610 \\
11 & 12,546 & 0.62730 \\
12 & 12,547 & 0.62735 \\
13 & 12,515 & 0.62575 \\
14 & 12,566 & 0.62830 \\
15 & 12,454 & 0.62270 \\
16 & 12,449 & 0.62245 \\
17 & 12,440 & 0.62200 \\
18 & 12,479 & 0.62395 \\
19 & 12,479 & 0.62395 \\
20 & 12,614 & 0.63070 \\
\hline
\end{tabular}

Table 2. Table of observations for large $\theta$

\begin{tabular}{ccc}
\hline Theta( $\boldsymbol{\theta})$ & Favourable Outcomes & Probability \\
\hline 1,000 & 12,677 & 0.63385 \\
1,050 & 12,572 & 0.62860 \\
1,100 & 12,596 & 0.62980 \\
1150 & 12,625 & 0.63125 \\
1,200 & 12,542 & 0.62710 \\
1,250 & 12,709 & 0.63545 \\
1,300 & 12,501 & 0.62505 \\
1,350 & 12,594 & 0.62970 \\
1,400 & 12,588 & 0.62940 \\
1,450 & 12,497 & 0.62485 \\
1,500 & 12,481 & 0.62405 \\
1,550 & 12,566 & 0.62830 \\
1,600 & 12,421 & 0.62105 \\
1,650 & 12,508 & 0.62540 \\
1,700 & 12,510 & 0.62550 \\
1,750 & 12,520 & 0.62600 \\
1,800 & 12,497 & 0.62485 \\
1,850 & 12,468 & 0.62340 \\
1,900 & 12,531 & 0.62655 \\
1,950 & 12,564 & 0.62820 \\
2,000 & 12,569 & 0.62845 \\
2,050 & 12,565 & 0.62825 \\
2,100 & 12,514 & 0.62570 \\
2,150 & 12,593 & 0.62965 \\
2,200 & 12,541 & 0.62705 \\
2,250 & 12,483 & 0.62415 \\
2,300 & 12,443 & 0.62215 \\
2,350 & 12,650 & 0.63250 \\
\hline & & \\
\hline
\end{tabular}




\begin{tabular}{lll}
\hline 2,400 & 12,515 & 0.62575 \\
2,450 & 12,460 & 0.62300 \\
2,500 & 12,578 & 0.62890 \\
2,550 & 12,474 & 0.62370 \\
2,600 & 12,625 & 0.63125 \\
2,650 & 12,668 & 0.63340 \\
2,700 & 12,517 & 0.62585 \\
2,750 & 12,494 & 0.62470 \\
2,800 & 12,502 & 0.62510 \\
2,850 & 12,557 & 0.62785 \\
2,900 & 12,490 & 0.62450 \\
2,950 & 12,449 & 0.62245 \\
3,000 & 12,689 & 0.63445 \\
\hline
\end{tabular}

\section{Concluding Remarks}

Through our theoretical investigation supplanted with experimental evidence, we have proved that the probability of obtaining real roots from a quadratic equation $A X^{2}+B X+C=0$ whose coefficients $A, B$, and $C$ are sampled from $\mathcal{U}(-\theta, \theta)$ is about $62.7 \%$. This is more than double the probability of $25.4 \%$ in the case of $U(0, \theta)$. Moreover, the expected value of the discriminant is positive in this case. In a scenario where one can only employ primitive arithmetic operations, the Newton-Raphson method for finding a root can be used to find one root, while the other can be obtained through addition or subtraction by twice the discriminant value.

\section{Appendix}

\section{Source Code}

The source code for the simulations, including the data, can be accessed at this repository: https://github.com/hungrybluedev/minus-theta-to-plus-theta.

Instructions on setting up the system and installing dependencies can be obtained from the README file. The Jupyter Notebooks provided can be run on any modern web browser.

\section{Expectation of $B^{2}$}

We make use of the law of the unconscious statistician (LOTUS) to calculate the expected value of $B^{2}$. For simplicity, we consider $B$ to be in the distribution $\mathcal{U}(0,1)$. We have already shown that this is equivalent to the $\mathcal{U}(+1,-1)$ case. Briefly, for continuous distributions, the LOTUS states that

$$
E[g(X)]=\int_{-\infty}^{+\infty} g(x) f_{X}(x) d x
$$

Therefore, for $B^{2}$, we have

$$
E\left[B^{2}\right]=\int_{0}^{1} b^{2} \cdot f_{B}(b) d b=\int_{0}^{1} b^{2} d b=\frac{1}{3}\left(1^{3}-0^{3}\right)=\frac{1}{3}
$$

\section{References}

[1] Nick Peterson (https://math.stackexchange.com/users/81839/nick-peterson), Probability that a quadratic equation has real roots, URL (version: 2020-09-08): https://math.stackexchange.com/q/3819057. The theoretical analysis is an expanded version of this answer. [accessed 9th Sept., 2020].

[2] Rozanov, Y. A. (1977). Probability Theory: A Concise Course, Dover Publications, Inc., N.Y., Dover Edition.

[3] D’Aurizio, J. (https://math.stackexchange.com/users/44121/jack-daurizio), Probability of a real root when coefficients are sampled from $\mathcal{U}(0,1)$ is $\approx 0.2544134190$ : Probability that a quadratic equation with random coefficients has real roots, URL (version: 2016-01-10): https://math.stackexchange.com/q/1607406. [accessed 9th Sept., 
2020].

[4] Felix Marin (https://math.stackexchange.com/users/85343/felix-marin), Probability that a quadratic equation has real roots, URL (version: 2020-09-09): https://math.stackexchange.com/q/3819074 - An interesting alternate solution using Iverson brackets (and integration) [accessed $9^{\text {th }}$ Sept., 2020].

[5] Gentle, J. E. (2009). Computational Statistics, Springer-Verlag, N.Y., Ist Ed. 